\title{
Enhancing Quality in Psychiatry with Psychiatrists (EQUIPP) - Results from a Pilot Study
}

\author{
Lea C. Watson, MD, MPH, \\ Department of Psychiatry, UNC School of Medicine, University of North Carolina at Chapel Hill, \\ Chapel Hill, North Carolina

\section{Denise A. Esserman, PhD,} \\ Division of General Medicine and Clinical Epidemiology, UNC School of Medicine, University of \\ North Carolina at Chapel Hill School, Chapel Hill, North Carolina; Department of Biostatistics, \\ UNC Gillings School of Public Health, University of North Carolina at Chapel Hill School, Chapel \\ Hill, North Carolina
}

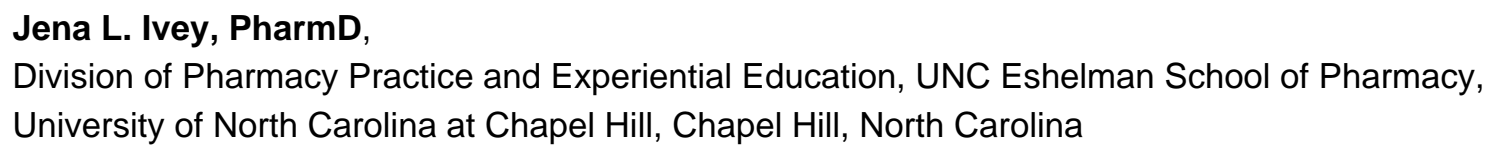

Carmen L. Lewis, MD, MPH,

Division of General Medicine and Clinical Epidemiology, UNC School of Medicine, University of North Carolina at Chapel Hill, Chapel Hill, North Carolina

\section{Richard Hansen, PhD,}

Department of Pharmacy Care Systems, Harrison School of Pharmacy, Auburn University, Auburn, Alabama

Morris Weinberger, PhD, and Department of Health Policy and Management, UNC Gillings School of Global Public Health, University of North Carolina at Chapel Hill School, Chapel Hill, North Carolina; Center for Health Services Research, Durham Veterans Affairs Medical Center, Durham, North Carolina

\section{Mary T. Roth, PharmD, MHS}

Division of Pharmaceutical Outcomes and Policy, UNC Eshelman School of Pharmacy, University of North Carolina at Chapel Hill, Chapel Hill, North Carolina

\section{Abstract}

\footnotetext{
(C) 2013 American College of Cardiology Foundation. Published by Elsevier Inc. All rights reserved.

Corresponding author: Lea C. Watson MD, MPH, Associate Professor of Psychiatry, CB \#7160, University of North Carolina at Chapel Hill School of Medicine, Chapel Hill, North Carolina, 27599-7160, phone: 919-966-5477, fax: 919-966-2220, lea_watson@med.unc.edu.

This work was presented at the American Association for Geriatric Psychiatry Annual Meeting, March 5 -8 2010, Savannah, Georgia. No disclosures to report.

Publisher's Disclaimer: This is a PDF file of an unedited manuscript that has been accepted for publication. As a service to our customers we are providing this early version of the manuscript. The manuscript will undergo copyediting, typesetting, and review of the resulting proof before it is published in its final citable form. Please note that during the production process errors may be discovered which could affect the content, and all legal disclaimers that apply to the journal pertain.
} 
Objectives-To pilot a pharmacist-led, patient centered medication management program.

Design-Prospective, single arm trial.

Setting-Academic geriatric psychiatry outpatient clinic.

Participants-Outpatients at least 65 years old, proxy available if demented, and on two or more psychiatric medications.

Intervention-A clinical pharmacist completed a baseline medication review and made evidence-based recommendations that were implemented by the pharmacist after discussion with the physician. The pharmacist made a minimum of monthly contact for 6 months to review medications and related issues.

Measurements-The primary outcome was the change in number of medication related problems over time (3 and 6 months) as defined by a predetermined classification system.

Results-The mean age of the 27 patients was 75 years, 10 of whom required a proxy to participate. On average, patients had 9 chronic conditions and were taking 14 medications. The mean number (SD; range) of medication related problems at baseline was $4.1(2.2 ; 0-8$,$) and at 3$ and 6 months were $3.6(2.4,0-9)$ and $3.4(2.1 ; 0-8)$, respectively. Most follow-up problems were new (80\% and $89 \%$ at 3 and 6 months, respectively).

Conclusion-Using a pharmacist to deliver a medication management program was feasible and addressed existing problems. However, new problems developed over a short interval (3-6 months), suggesting that ongoing intervention is required.

\section{Keywords}

medications; pharmacist; quality

\section{OBJECTIVES}

Compared to younger adults, older adults ( $\ 65$ years) take more medications, have more medication-related negative outcomes, and utilize more health services (1). Older adults with psychiatric disease have even more pronounced risks because psychoactive drugs are disproportionately associated with delirium, falls, and adverse drug interactions (2). Psychiatric settings are not typically equipped to provide global medication review and management, despite treating older patients with extensive comorbidity and complex medication regimens.

Pharmacist-led interventions have improved the quality of medication use in many settings(3). Pharmacists can improve patients' outcomes by monitoring symptoms, counseling, resolving medication-related problems, encouraging adherence to medications, and facilitating communication with physicians. To our knowledge, such interventions have never been evaluated among older psychiatric outpatients, a vulnerable and medically complex group of patients. The purpose of this study was to pilot test a novel pharmacist-led medication management program that specifically targets older psychiatric outpatients. 


\section{METHODS}

This pilot study tested a 6-month, pharmacist-led medication management program on medication-related problems at 3 and 6 months. The details of this study have been described previously (4) and are summarized here. The UNC Biomedical Institutional Review Board, Chapel Hill, North Carolina, approved the study.

\section{Setting and participants}

Participants were recruited from an academic geriatric psychiatry outpatient clinic.

Eligibility criteria included: current enrollment in the clinic for any diagnosis, age $\ 65$ years, English speaking, not in hospice care, and being prescribed $\geq 2$ central nervous system (CNS)-active medications. In addition, a proxy had to be available if the patient had a diagnosis of dementia or screened positive for cognitive impairment on a 6-item cognitive screen(5). The rationale for involving a proxy was not only to assure appropriate consent, but also to target the person most responsible for managing the patient's medications.

\section{Data Collection Process and Measures}

Patients were evaluated at baseline, 3 months and 6 months. Informed consent was obtained, including assent from patients when a proxy was indicated. The baseline visit consisted of an interview with the research assistant (RA) and a comprehensive medication review with the clinical pharmacist. Detailed methods are reported elsewhere (4), but included chartbased information on psychiatric diagnoses, health care utilization, and medication adherence determined by a direct pharmacist clinical assessment (6). When a proxy consented, the proxy reported on the patients' medication adherence.

\section{Intervention}

The clinical pharmacist initiated a multi-faceted medication evaluation and management intervention that involved close collaboration with the patient's primary physician. The medication evaluation and intervention occurred face-to-face at baseline; subsequent evaluations and interventions occurred via telephone. The intervention was patient-centered and took into account patient preferences for care (7).

Medication-related problems-The clinical pharmacist's assessments were guided by a list of medication-related problems developed by study investigators following an extensive review of the literature and subsequent refinement of previous work(4). The potential medication-related problems are described in the footnote to Figure 1. This approach to formulating assessments of quality medication use was developed specifically to be practical, comprehensive, and amenable to clinical practice. Upon completion of the comprehensive medication review and medical record review, the clinical pharmacist formulated a written assessment of the quality of medication use along with a proposed plan.

Recommendations and plan of care-Recommendations were communicated to the primary care physician and other relevant prescribers. The preferred method of communication was via phone; however, if the pharmacist could not reach the physician by phone following several attempts, the assessment and plan were faxed to the physician's 
office. The faxed note requested that the physician indicate his/her acceptance, rejection, or revision to the proposed plan and return the fax or phone call to the pharmacist. The final plan for addressing medication-related problems was developed collaboratively with the provider to ensure coordination and continuity of care.

All patients/proxies received a call from the clinical pharmacist informing them of the assessment and plan for improving their medication use. Additionally, all physicianapproved medication changes were discussed in detail with the patient/proxy, including how changes would be made. For example, if the change involved purchase of a nonprescription medication, the pharmacist instructed the patient/proxy as to where they could obtain the new product, what exactly to look for, and instructions regarding its use. The clinical pharmacist also provided education, including the purpose, proper use, anticipated response to therapy, and potential adverse effects of each medication. Regardless of who was responsible for implementing the medication changes, the clinical pharmacist provided the educational component by phone. All patients/proxies were mailed a written summary of the medication assessment and plan. When mailing the written summary, the clinical pharmacist also provided the patient with a personal medication record. The personal medication record, developed by study investigators, contained an up-to-date listing of all medications the person was to be taking (prescription, nonprescription, complementary and alternative), including strength, frequency, instructions for use, and indication.

A key component of the intervention was documentation of the comprehensive medication review, with final assessment and plan. This documentation took the form of a traditional SOAP (subjective, objective, assessment, plan) note and was either entered directly into the patient's electronic medical record, or, if the patient's primary care records were located elsewhere, faxed to the physician's office. Moreover, documentation included attention to medication reconciliation among all providers; the clinical pharmacist updated the medication records for all patients in the electronic medical record system and, when faxing notes to physician offices, called attention to the need to update the patient's medication list.

Follow-up-Finally, the clinical pharmacist followed the patient for 6 months, providing periodic medication evaluation and management by phone. All patients/proxies received monthly follow-up phone calls to evaluate the potential for ongoing or new medicationrelated problems and address any medication-related questions. Where needed and in collaboration with the physician, the clinical pharmacist intervened to resolve medicationrelated problems over the 6-month period. All encounters with a patient were documented in the study database.

\section{Outcome assessments}

A second pharmacist provided independent assessments of medication-related problems at 3 and 6 months. Although the second pharmacist did not communicate with the clinical pharmacist when assessing medication-related problems, she did have access to the clinical pharmacist's assessment and recommendations in the electronic medical record. An adjudication process was used by the study team to assure consensus when applying the medication-related problem framework and making recommendations. A third pharmacist 
and one physician also reviewed a random sample of patient records at all three visits to confirm the appropriate use of the framework.

\section{Data Analysis}

Descriptive statistics, means, standard deviations (SD) and ranges for continuous data and frequencies and percentages for categorical data, were calculated. Poisson mixed models (random intercept) were used to assess a trend over time in the primary outcome of medication related problems at 3 and 6 months.

\section{RESULTS}

Of 173 adults assessed for inclusion, 146 were not eligible $(n=63)$, reachable $(n=25)$, or interested $(n=58)$ in participating. Twenty-seven patients completed the baseline interview and one patient died shortly after enrollment due to unanticipated complications from prostate cancer, leaving 26 participants who completed the 3 and 6 month interviews. Of the baseline patients, 10 (37\%) were cognitively impaired, requiring proxies to take part in medication discussions on their behalf. Patients were on average 75 years of age (SD 8.1), mostly female $(n=17,63 \%)$, white $(n=20,74 \%)$, educated ( $93 \%$ had at least HS degree), and had multiple chronic conditions (mean 8.7, SD 2.8). With regard to psychiatric diagnoses documented in the chart, 20 (74\%) listed depression, 9 (33\%) anxiety, 9 (33\%) dementia and 3 (11\%) bipolar disorder (these were not mutually exclusive). The mean number of medications was 14.0 (SD 4.7) (including over-the-counter and herbal medications) and each participant had a mean of 3 (SD 1.0) prescribers.

At baseline each participant had a mean of 4.1 (SD 2.2) medication-related problems. There was not a significant decrease in overall problems over time (3 months: mean 3.6, SD 2.4; 6 months: mean 3.4, SD 2.1). Baseline problems tended to resolve (3 months: mean 0.7, SD 1.0; 6 months: mean 0.4, SD 0.9), but were replaced by new problems at the 3 (mean 2.0, SD 2.0) and 6 month (mean 3.0, SD 2.1) visits. Figure 1 displays the breakdown of these problems by category over time. Suboptimal medication use, suboptimal dose and potential problem were most prevalent at baseline, with potential problem and suboptimal drug improving over time. Non-adherence, while seen in $14 \%$ of participants at baseline, rose to $34 \%$ and $33 \%$ at 3 and 6 months respectively, while the total number of medications did not change over time. Visits to the Emergency Department or transfers to higher levels of care did not change relative to the self-reported 6 months prior to the intervention. During follow-up phone calls with the patients, only one potential problem was identified regarding a duplicate order for a patient who had a transition in care.

\section{CONCLUSIONS}

This pilot study tested a novel medication management program designed to improve the quality of medication use in vulnerable older adults who receive outpatient care from psychiatrists. On the primary outcome of medication-related problems over time, there was no change in the overall number of medication related problems at 3 or 6 months. As a pilot study, we were not powered to detect this difference. There was no change in health service 
utilization or transfers to higher levels of care compared to the 6 months prior to the study. The intervention proved feasible in the psychiatric clinic.

This study extends a framework for defining medication related problems in older adults and provides preliminary data on a novel intervention for addressing them. We learned that despite a comprehensive patient-centered evaluation and initial improvement in medication quality following one visit, medication-related problems were present six months later. One likely possibility is that new and different problems arose over time, rather than the persistence of problems identified at baseline. It may be that a control group would have developed more problems over time and that the unchanged number of problems as reflected in our outcome is in fact a sign of stability. A longer follow-up may have been required to observe a reduction in the number of medication-related problems identified. Alternatively, because monthly follow-up was done by phone, interventions by the clinical pharmacist may have had less impact compared to face-to-face intervention at baseline, where direct observation of medications and measures of adherence could be verified. The nature of the phone calls was brief and not standardized in this study, mostly offering an opportunity for the patient to ask any questions about their medications, and questions were few. The categories of problems that occurred over time, while limited in power, offer hints as well, showing that adherence to medications worsened over time but use of suboptimal medications decreased over time balancing out the total number of problems. Possible reasons for non-adherence include that new medications were introduced, that there was a different pharmacist assessing for adherence for follow-up visits and that these were done by phone instead of face-to-face, and it is possible that patients became more fearful of their medications when the pharmacist described them in detail.

This study has the inherent limitations of a pilot design, specifically in its lack of a control group and small size. In addition, despite our attempt to provide a systematic adjudication regarding the number of medication problems over time, the second pharmacist may have identified problems with a different level of scrutiny, reflecting a potential detection bias.

Despite these limitations, this report provides important findings. The patients in this study had multiple chronic diseases and were on an average of 14 medications, highlighting a high burden of illness. While aging experts have long realized the special needs of complex patients and the potential benefits of pharmacist-delivered interventions (2), this is the first report to our knowledge of such a comprehensive and patient-centered medication management program in geriatric psychiatry. Using a clinical pharmacist to deliver a medication management program in this specialty setting was feasible and addressed existing problems. However, new problems developed over a short interval (3-6 months), suggesting that ongoing intervention is required.

\section{Acknowledgments}

Sources of support:

Dr. Watson was supported by a UNC Investments for the Future Award, School of Medicine, University of North Carolina at Chapel Hill, and by a Multidisciplinary Clinical Research Career Development Award (1K12HD052191) from the National Institutes of Health. Dr. Roth was supported by a K23 Mentored Career Development Award from the National Institute on Aging (5K23AG024229). Drs. Esserman and Weinberger 
received funding from the National Institutes of Health (CTSA UL1RR025747). Dr. Hansen was supported by a National Center for Research Resources award (KL2 RR025746). Dr. Lewis was supported by a K07 Mentored Career Development Award (5K07CA104128) from the National Cancer Institute.

The authors would like to thank Robin Zook PharmD for her thoughtful care of study participants in her role as the second pharmacist. The authors also wish to thank Ryan Owenby, PharmD for his assistance in project management and data entry.

\section{References}

1. Gurwitz JH, Field TS, Harrold LR, et al. Incidence and preventability of adverse drug events among older persons in the ambulatory setting. JAMA. 2003; 289:1107-1116. [PubMed: 12622580]

2. Shrank WH, Polinski JM, Avorn J. Quality indicators for medication use in vulnerable elders. J Am Geriatr Soc. 2007; 55 (Suppl 2):S373-382. [PubMed: 17910560]

3. Lu CY, Ross-Degnan D, Soumerai SB, et al. Interventions designed to improve the quality and efficiency of medication use in managed care: a critical review of the literature - 2001-2007. BMC Health Services Research. 2008; 8:75. [PubMed: 18394200]

4. Roth MT, Watson LC, Esserman DA, et al. Methodology of a pilot study to improve the quality of medication use in older adults: Enhancing Quality in Psychiatry Using Pharmacists (EQUIPP). The American Journal of Geriatric Pharmacotherapy. 2009; 7:362-372. [PubMed: 20129257]

5. Callahan CM, Unverzagt FW, Hui SL, et al. A six-item screener to identify cognitive impairment among potential subjects for clinical research. Medical Care. 2002; 40:771-781. [PubMed: 12218768]

6. Roth MT, Ivey JL. Self-reported medication use in community-residing older adults: A pilot study. The American Journal of Geriatric Pharmacotherapy. 2005; 3:196-204. [PubMed: 16257822]

7. Chewning B, Sleath B. Medication decision-making and management: a client-centered model. Social Science \& Medicine. 1996; 42:389-398. [PubMed: 8658233] 


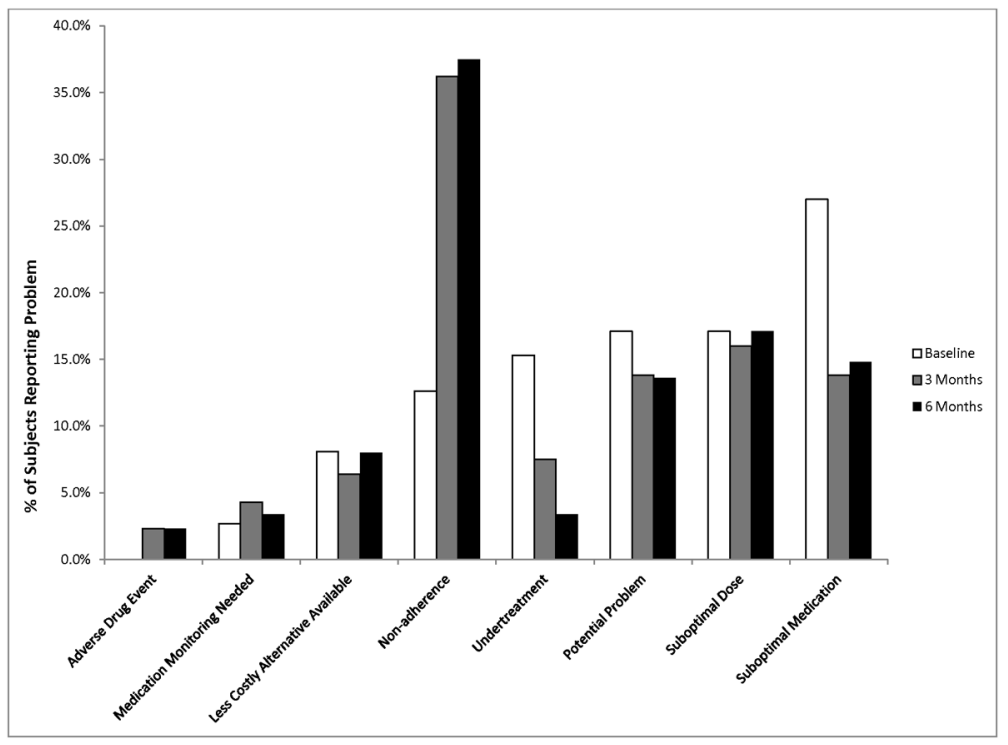

Figure 1.

Distribution of Medication-Related Problems by Type of Problem Over Time

\begin{tabular}{|c|c|}
\hline Medication-related Problems & Definition \\
\hline Suboptimal medication & $\begin{array}{l}\text { The individual is receiving a drug that has no indication, is not effective, or is } \\
\text { potentially not safe (i.e., risk of using drug outweighs benefit). }\end{array}$ \\
\hline Suboptimal dose & $\begin{array}{l}\text { The individual is taking an appropriate medication, but the dose is not optimal to } \\
\text { achieve desired response, or has the potential for harm. }\end{array}$ \\
\hline Adverse drug events & $\begin{array}{l}\text { The individual is experiencing adverse consequences attributed to a drug or the } \\
\text { inappropriate use of a drug. }\end{array}$ \\
\hline Nonadherence & $\begin{array}{l}\text { The individual has not filled a prescription, is not taking a drug, or is not using a } \\
\text { drug as prescribed, whether intentional or unintentional (determined by direct } \\
\text { pharmacist clinical assessment). }\end{array}$ \\
\hline Less costly alternative available & $\begin{array}{l}\text { The individual is prescribed a medication, for which a less costly, equally effective } \\
\text { and safe drug is available, and preferred by the patient, but the patient is receiving a } \\
\text { more expensive product; or the patient could benefit from prescription assistance, } \\
\text { but is not receiving the benefit and desires to. }\end{array}$ \\
\hline Undertreatment & $\begin{array}{l}\text { The individual has a medical condition that would benefit from drug therapy (clear } \\
\text { indication) and the patient has no contraindications to the drug, but the drug was not } \\
\text { prescribed. }\end{array}$ \\
\hline Medication monitoring needed & $\begin{array}{l}\text { The individual is receiving a drug and monitoring is required to assess response to } \\
\text { therapy or prevent harm, but has not been done. }\end{array}$ \\
\hline Potential problem & $\begin{array}{l}\text { There is a potential medication-related problem warranting physician referral and } \\
\text { further evaluation. The potential problem cannot be classified as a medication- } \\
\text { related problem without further physician evaluation of the problem. }\end{array}$ \\
\hline
\end{tabular}

